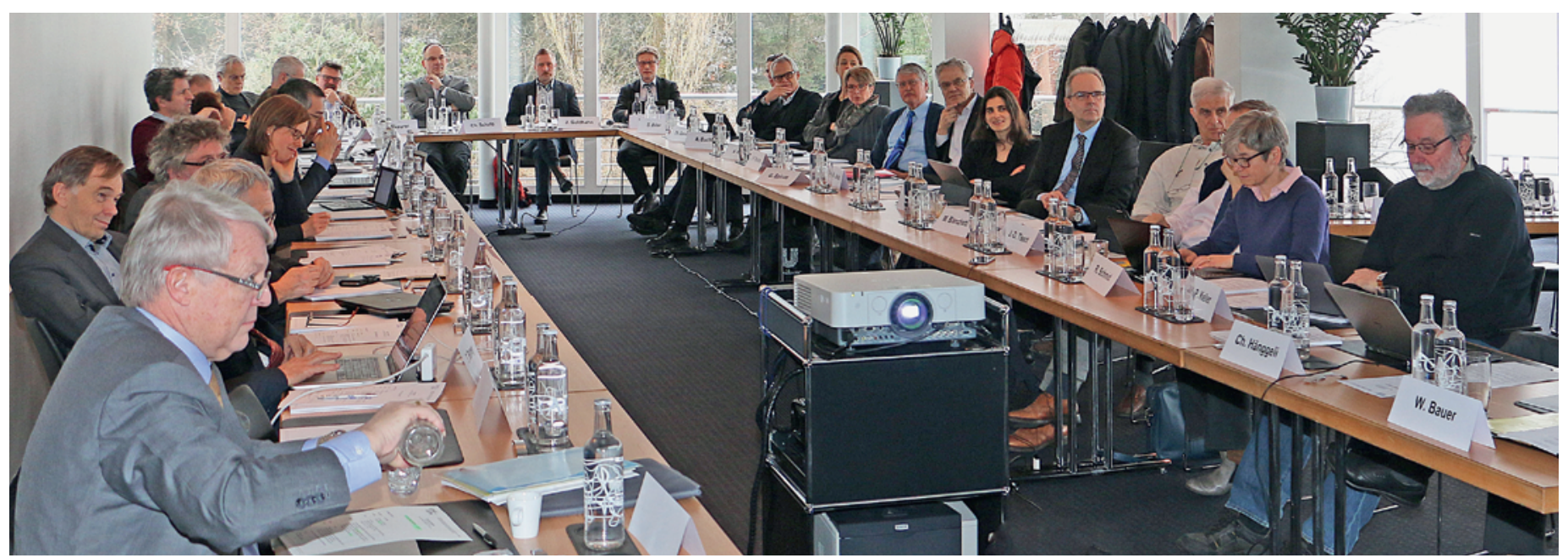

La Journée de réflexion réunit des experts de la formation médicale.

\title{
Donner des impulsions menant à des résultats
}

\section{Bruno Kesseli}

Dr méd. et lic. phil., rédacteur en chef

La traditionnelle Journée de réflexion de l'ISFM et du Collège des Doyens a offert cette année également des exposés passionnants; elle a donné à des experts de la formation médicale postgraduée et continue l'occasion d'échanger de manière intensive.

Les organisateurs sont d'accord entre eux. Henri Bounameaux, président du Collège des Doyens, et Werner Bauer, président de l'ISFM, soulignent dans leur allocution de bienvenue l'importance de la Journée de réflexion en tant que forum d'information et de discussion pour les acteurs de la formation médicale. Un coup d'œil sur la liste impressionnante des participants confirme leur présence en grand nombre.

\section{Davantage que des idées librement échangées}

Werner Bauer insiste sur le fait qu'il ne s'agit pas seulement de s'exprimer librement car cette journée vise aussi à donner des impulsions susceptibles de mener à des résultats concrets. Henri Bounameaux abonde dans ce sens avec un exemple. Dans le cadre de la "Journée 2016», le Collège des Doyens avait adopté une prise de position sur l'organisation des études de méde- cine. Publiée dans le BMS [1], cette prise de position a influencé de manière perceptible la discussion sur les changements à effectuer dans la formation médicale. Henri Bounameaux mentionne également d'autres engagements du Collège des Doyens, par exemple dans les groupes de travail de l'Office fédéral de la santé publique (OFSP) et de l'Académie suisse des sciences médicales (ASSM).

\section{Journée de réflexion}

La Journée de réflexion est ancrée depuis des années dans le calendrier des rencontres en lien avec la formation médicale. Elle est organisée par l'Institut suisse pour la formation médicale postgraduée et continue (ISFM) et par le Collège des Doyens des facultés de médecine suisses. Cette année, elle a eu lieu les 21 et 22 janvier à Lucerne. 


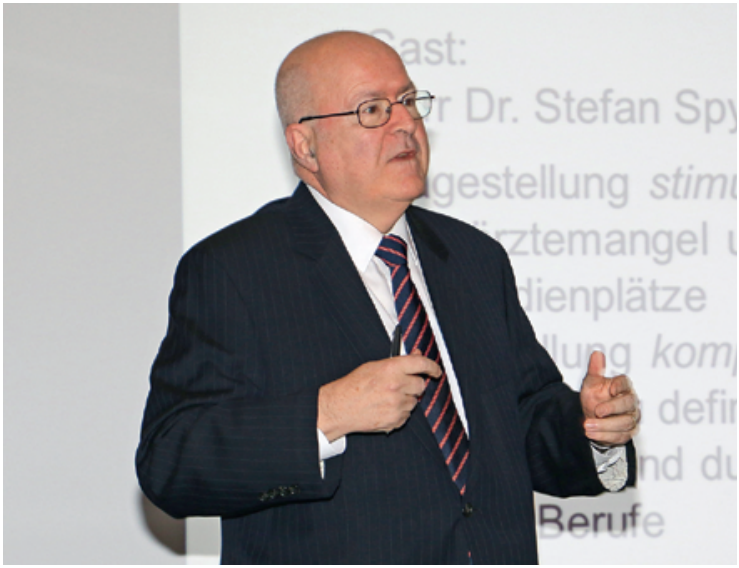

Werner Bauer, co-organisateur, souhaite la bienvenue et présente rapidement les objectifs de la rencontre.

\section{Prise en charge optimale de la population en matière de soins}

Après que Christoph Hänggeli, directeur de l'ISFM, a récapitulé les tâches et activités variées de l'Institut dans un flash info compact, Mauro Poggia s'exprime sur la Couverture médicale optimale de la population suisse: problèmes et pistes de solution. Le directeur de la santé publique du canton de Genève rappelle tout d'abord les fondamentaux, certes pas banals, de la prise en charge médicale dans notre pays. La Confédération dispose d'un système de santé particulièrement complexe qui, en dépit de nombreux avantages incontestés, parvient lentement à ses limites notamment en matière de financement. Parmi les acteurs impliqués, seuls les cantons ont un véritable intérêt à limiter les coûts de santé. De manière générale, on observe une tendance à la déresponsabilisation des parties prenantes. Quant à la Confédération, Mauro Poggia détecte une certaine passivité dans son rôle de législateur, il constate un manque de transparence dans le domaine des données et il définit le pilotage du système comme inefficace. A titre d'exemples de déresponsabilisation, il mentionne la tendance à l'augmentation des volumes par les fournisseurs de prestations, la compensation insuffisante des risques chez les assureurs, la surconsommation de soins par les assurés et l'idéalisation du modèle de concurrence entre la Confédération et les cantons.

\section{Caisse de compensation comme solution?}

Une réforme du système est inévitable face aux défis connus à venir comme la hausse du nombre de patients âgés polymorbides. Mauro Poggia a des idées claires sur les grandes lignes de cette réforme. Il envisage un modèle offrant aux cantons la possibilité d'introduire une "caisse de compensation». Celle-ci fixerait les primes et les encaisserait, tandis que les caissesmaladie continueraient de contrôler les factures et le suivi des assurés. Il en résulterait une uniformisation des primes à l'intérieur de chaque canton, ce qui supprimerait la chasse aux bons risques. Les défenseurs de ce modèle attendent un effet stabilisateur sur les primes et les tarifs. Naturellement, les discussions menées dans les médias montrent que cette idée suscite la controverse quant à la question de savoir si elle est réaliste; les débats devraient se concrétiser cet été dans le cadre d'une initiative populaire fédérale.

Dans la discussion qui s'en est suivie, Mauro Poggia envisage aussi d'autres mesures comme la régulation locale de l'installation des médecins. Dans le contexte actuel, il est d'avis que la profession médicale ne peut pas être totalement libérale. Werner Bauer pose la question de l'impact de ces considérations centrées sur

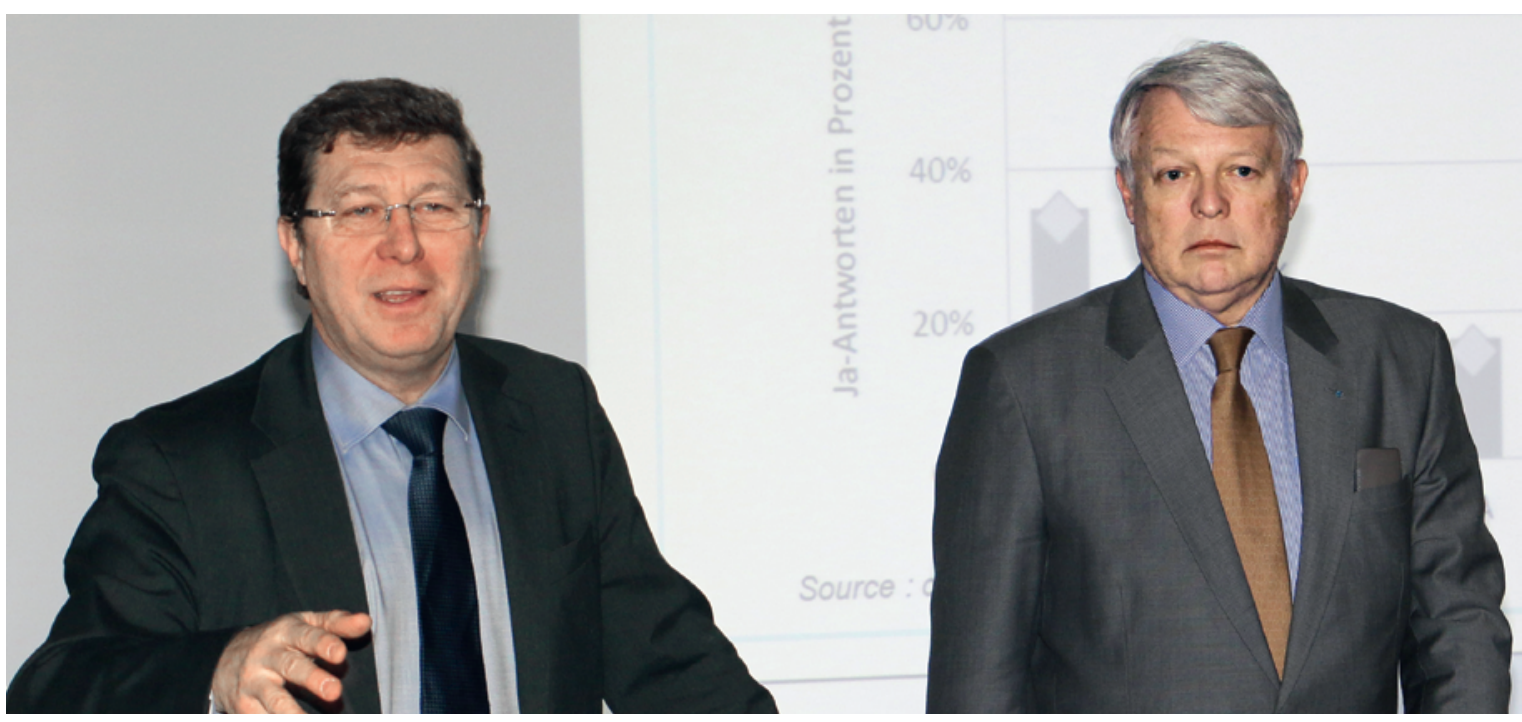

Mauro Poggia, intervenant, et Henri Bounameaux, co-organisateur. 
les coûts pour la formation médicale et Henri Bounameaux rappelle que les nombreuses tâches de coordination des médecins de premier recours sont généralement mal rémunérées. Mauro Poggia souligne que, pour ces domaines, il faut mettre suffisamment de moyens à disposition car sinon les dimensions essentielles de la médecine se perdront. Mais les médecins doivent aussi être prêts à accepter de nouvelles professions d'appui, justement dans la coordination des prestations de santé.

\section{Pilotage de la prise en charge médicale}

Le deuxième exposé de la journée intitulé Pilotage des forces médicales actives est présenté par Stefan Spycher, vice-directeur de l'OFSP. Dans son introduction, Werner Bauer est d'avis que le corps médical devrait discuter activement de ce sujet et il présente le «modèle PaysBas", vu aujourd'hui par beaucoup comme un exemple pour la Suisse. Ce modèle vise à évaluer le besoin futur en médecins sur la base des effectifs actuels et de l'évolution prévue. Dans ce contexte, de nombreux facteurs sont pris en compte, d'ordre démographique, épidémiologique et technologique.

Stefan Spycher identifie cinq problèmes importants relatifs à l'approvisionnement médical en Suisse:

- Pas assez d'étudiants / dépendance élevée face à l'étranger

- Age élevé à la fin de la formation postgraduée

- L'offre de postes de formation postgraduée ne correspond pas aux besoins

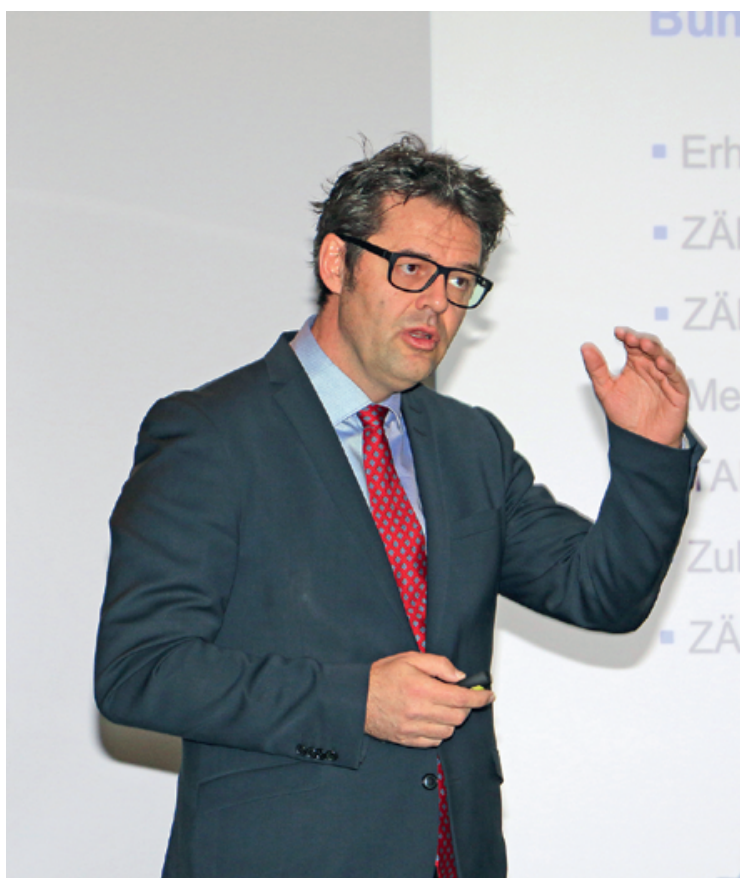

Stefan Spycher s'exprime sur les questions de pilotage.
- Installation non régulée dans le domaine ambulatoire-avant tout de médecins étrangers, ce qui peut augmenter les coûts pour les patients sans un avantage supplémentaire clair

- Malgré le nombre suffisant de médecins, difficultés de remettre un cabinet en région rurale

La Confédération a déjà lancé différentes initiatives visant à apporter des solutions à ces problèmes. Stefan Spycher mentionne l'augmentation du nombre d'étudiants et différents groupes de travail de la plate-forme "Avenir de la formation médicale», dont un qui traite spécifiquement des questions de coordination de la formation médicale postgraduée. Il estime que le pilotage des admissions à pratiquer à la charge de l'assurance obligatoire et la réduction des incitatifs négatifs grâce à la révision du TARMED font également partie de ce paquet. Sa conclusion est que la planification des forces actives peut contribuer à résoudre ces problèmes mais qu'il faut procéder à différents réglages si l'on veut maintenir ou encore améliorer la qualité élevée de la formation prégraduée et postgraduée et celle des soins médicaux en Suisse.

\section{Aucun remède miracle}

La discussion montre clairement que personne n'a de recette miracle qui permettrait de maîtriser intégralement les défis actuels et futurs. Werner Bauer rappelle un point qui lui paraît essentiel pour développer des propositions de solution. Il est persuadé que le profil du médecin de famille va changer et que les acteurs concernés doivent absolument en tenir compte dans leurs réflexions. Stefan Spycher approuve l'intervention de Werner Bauer et garantit que cette question est effectivement traitée dans différents comités d'experts.

La discussion sur les questions de pilotage prend beaucoup d'espace. Johann Steurer, directeur du centre Horten pour la recherche orientée sur la pratique et le transfert des connaissances de l'université de Zurich, plaide comme il l'a déjà fait à d'autres occasions [2] pour le début de la spécialisation dans les études de médecine déjà après l'acquisition du bachelor. Différents intervenants soulignent l'importance de la question financière dans le pilotage. Rainer Weber, doyen de la faculté de médecine de l'université de Zurich, mentionne un autre aspect, à savoir qu'il faudrait aussi parler de surplus de médecins car c'est clairement le cas dans certaines spécialisations. Il précise que le problème est en outre devenu plus aigu par l'obligation d'admettre tous les spécialistes de l'Espace européen, aussi ceux qui ont éventuellement reçu une formation prégraduée et postgraduée insuffisante. 


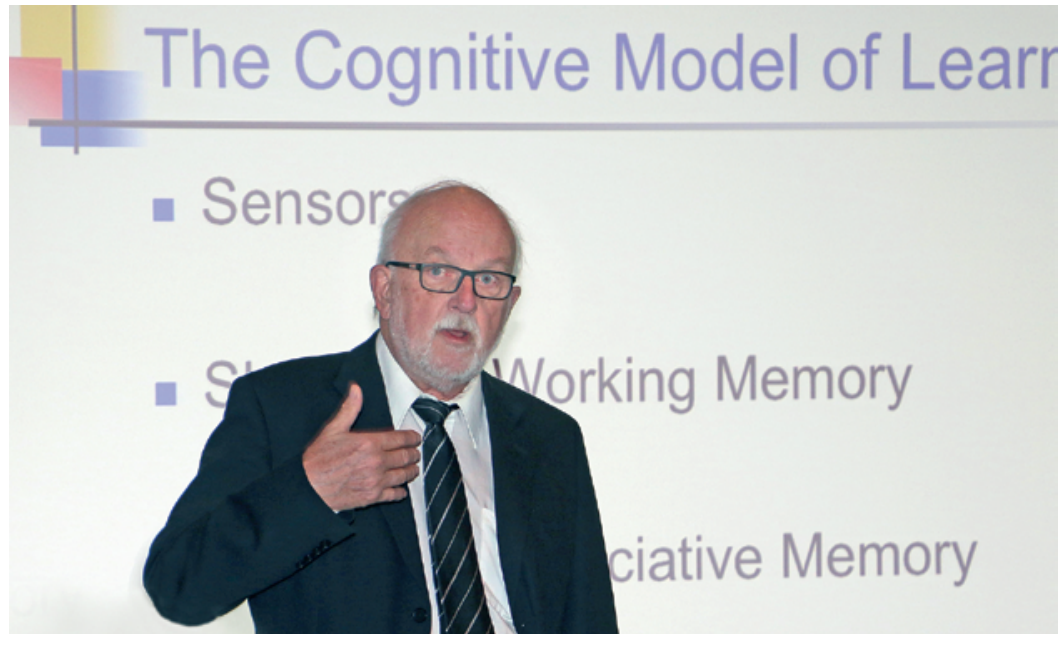

Façonner activement les processus d'apprentissage: Geoff Norman.

\section{Le cerveau fonctionne par association}

Dans son exposé How people learn and how this affects teaching and curriculum, Geoff Norman donne un aperçu fascinant de la manière avec laquelle l'être humain apprend. Physicien nucléaire, psychologue et professeur émérite d'épidémiologie clinique et de biostatistique de l'université McMaster au Canada, il consacre son travail à la formation médicale dont il a étudié les instruments de manière scientifique et pour le développement de laquelle il a fourni d'importantes contributions.

A l'aide de différents exemples, Geoff Norman démontre clairement que la métaphore de l'ordinateur pour définir le cerveau humain est trop réductrice. Sur des aspects essentiels, la manière de fonctionner du cerveau se distingue fondamentalement de l'ordinateur. Il est plus lent et sa capacité à traiter des données ainsi que sa mémoire vive sont bien plus petites. En revanche, sa capacité d'enregistrement atteint des dimensions presque inimaginables. Dans ces conditions, il est étonnant que nous soyons en mesure de parvenir, pour certaines questions, à des résultats aussi bons qu'un ordinateur. Si l'on nous demande par exemple quelle est la capitale de la France, nous avons immédiatement la bonne réponse et nous sommes conscients que nous le savons. Par contre, si l'on nous demande quelle est la capitale du Tadjikistan, nous savons aussi immédiatement que nous ne connaissons pas la réponse (du moins beaucoup d'entre nous).

Ces résultats sont possibles uniquement parce que le cerveau se branche sur notre mémoire associative et ne questionne pas notre mémoire de manière séquentielle comme un ordinateur. Inversement, nous n'emmagasinons pas les informations de manière isolée lorsque nous apprenons mais les nouvelles connaissances sont toujours mises en lien avec des connaissances déjà existantes. Saisir la signification est ainsi centrale pour le processus d'apprentissage. Par exemple, les professionnels du jeu d'échecs sont en mesure, en comparaison avec les joueurs amateurs, d'envisager un nombre varié de positions réelles de jeu lorsqu'ils le voient pendant cinq secondes. Par contre, lorsque les pions sont placés au hasard et «sans signification» sur l'échiquier, les professionnels ne s'en sortent pas mieux que les amateurs parce qu'ils ne peuvent pas relier l'image à leurs connaissances antérieures.

De telles constatations sont également importantes pour la formation médicale. Il faudrait organiser les processus d'apprentissage de manière active, enseigner si possible les principes à l'aune de problèmes concrets et recourir à des analogies tirées de la vie quotidienne pour les comprendre. En s'appuyant sur différentes études, Geoff Norman fournit la preuve que cette manière d'enseigner conduit à de meilleurs résultats que «l'enseignement séquentiel» qui consiste à expliquer tout d'abord les principes puis à donner des exemples.

\section{De la réflexion à l'action}

A la fin de la rencontre, le bloc réservé aux «questions ouvertes de la formation médicale» a suscité une discussion animée à la suite de laquelle Werner Bauer a établi une liste possible des To do intitulée «De la réflexion à l'action». Par prudence, il a accompagné certaines affirmations d'un point d'interrogation. Les principales suggestions concernent notamment la mise en valeur du Teaching dans les hôpitaux, le passage en revue du nombre de postes de formation postgraduée, le remaniement du catalogue des objectifs de formation ou la mise en place d'une structure davantage en réseau pour la formation postgraduée. De même, il inscrit sur la liste la question de savoir si d'autres professionnels (et lesquels) pourraient assumer des activités médicales, la garantie du financement de la formation postgraduée, l'alliance de la formation postgraduée avec une activité scientifique et le développement de tracks dans les études.

Les experts présents sont rentrés chez eux avec le sentiment d'avoir fait le plein d'idées. On est curieux de voir si des solutions concrètes à certains problèmes et questionnements seront présentées déjà à la prochaine Journée.

\section{Références}

1 Bounameaux H, Eggli P, Gasser T, Tissot JD, Weber R. Statement regarding medical education in Switzerland. Bulletin des médecins suisses. 2016;97(12-13):461-2.

2 Kesseli B. Se préparer efficacement aux défis de demain. Bulletin des médecins suisses. 2015;96(45):1638-41. 